Trauma Berufskrankh 2018 $\cdot 20: 230-236$ https://doi.org/10.1007/s10039-018-0400-0 Online publiziert: 5 . September 2018

(c) Der/die Autor(en) 2018

CrossMark

\author{
C. von Rüden ${ }^{1,2} \cdot$ S. Samsami ${ }^{2} \cdot$ R. Pätzold' $\cdot$ P. Augat ${ }^{2,3}$ \\ ${ }^{1}$ Abteilung Unfallchirurgie, BG Unfallklinik Murnau, Murnau, Deutschland \\ ${ }^{2}$ Institut für Biomechanik, BG Unfallklinik Murnau, Murnau, Deutschland \\ ${ }^{3}$ Institut für Biomechanik, Paracelsus Medizinische Privatuniversität, Salzburg, Österreich
}

\title{
Proximale Tibiafrakturen
}

\section{Klassifikationen und biomechanische Grundlagen - ein Update}

\begin{abstract}
Die dreidimensionale CT-basierte Bildgebung ermöglicht eine präzise Analyse der Frakturmorphologie proximaler Tibiafrakturen. Auf der Basis einer akkuraten Darstellung der Frakturfragmente lässt sich die osteosynthetische Stabilisierung sorgfältig planen. Die konsequente Anwendung biomechanischer Prinzipien erlaubt die Wiederherstellung der Gelenkfunktion. Schließlich ermöglicht die Berücksichtigung der Ergebnisse zahlreicher biomechanischer Untersuchungen eine zielgerichtete Auswahl des geeigneten Osteosyntheseverfahrens und -materials.
\end{abstract}

Proximale Tibiafrakturen gehören zu den herausforderndsten Frakturen und sind mit einer hohen Inzidenz an Komplikationen und posttraumatischer Arthrose verbunden. Wesentlicher Faktor für die Vermeidung von Komplikationen ist die Wiederherstellung der optimalen Gelenkfunktion. Hierfür muss die Gelenkstabilität durch eine korrekte Einstellung der auf das Gelenk einwirkenden Kräfte und die vollständige anatomische Reposition der Gelenkflächen gesichert werden. Um dieses Ziel zu erreichen, sind eine optimale operative Maßnahme und eine sorgfältige und zielgerichtete postoperative Rehabilitation notwendig. Die präzise chirurgische Therapie ist allerdings abhängig von einer optimalen präoperativen Planung, die das richtige Verständnis für die Frakturmorphologie ermöglicht. Hierzu ist es notwendig, die Klassifikationen und deren Implikationen für die Planung des operativen Vorgehens zu kennen, insbesondere durch Berücksichtigung moderner dreidimensionaler Bildgebungsverfahren. Weiterhin ist die Kenntnis der mechanischen Verhältnisse am Kniegelenk wichtig, um die vorhandenen Osteosynthesetechniken adäquat anwenden zu können.

\section{„Klassische“" Klassifikationen}

In den vergangenen Jahrzehnten wurden verschiedene Klassifikationen für proximale Tibiafrakturen mit dem Ziel entwickelt, eine optimale Behandlungsstrategie finden zu können und dadurch letztlich den klinischen Langzeitverlauf $\mathrm{zu}$ verbessern. Die verschiedenen Klassifikationssysteme blieben allerdings relativ subjektiv, da sie auf der diagnostischen Erfahrung und v. a. der Art des zugrunde liegenden bildgebenden Verfahrens basierten. Als bildgebende Verfahren für bisherige Klassifikationen dienten das konventionelle Röntgen, die Computertomographie (CT) und die Magnetresonanztomographie (MRT), wobei stets die Intra- und Interbeobachterreliabilität zu beachten sind [24]. Während infolge technischer Fortschritte die genannten Untersuchungsverfahren das Frakturmuster präoperativ immer detailgenauer darstellen, besteht nach wie vor die Notwendigkeit zu überprüfen, ob diese Darstellungsverfahren eine ausreichend genaue Einteilung der verschiedenen Frakturkonfigurationen ermöglichen, um die Planung der operativen Maßnahme sinnvoll zu unterstützen und das Nachbehandlungsregime positiv beeinflussen zu können [36]. Von allen bisher vorliegenden Klassifi- kationen für proximale Tibiafrakturen sind nur wenige hinsichtlich ihrer Reliabilität untersucht und noch weniger miteinander verglichen worden. Die weltweit am häufigsten angewendete Schatzker-Klassifikation [39] und die im deutschsprachigen Raum weit verbreitete $\mathrm{AO}$ (Arbeitsgemeinschaft Osteosynthesefragen)-Klassifikation ( $\bullet$ Abb. 1; $[30,38])$ sind umfangreich untersucht und beschrieben worden und gelten als Standardklassifikationen für proximale Tibiafrakturen. Während beide Systeme die höchste Prävalenz zu haben scheinen, bleibt die Frage, ob sie ausreichend effektiv sind, alle Frakturmuster genügend abzubilden, insbesondere hinsichtlich der daraus abzuleitenden Therapieentscheidung. Neben anderen historischen Klassifikationen wie der Duparc- und Ficat-Klassifikation [9], der Hohl- und Luck-Klassifikation [21] oder der Moore-Klassifikation für Tibiakopfluxationsfrakturen (- Abb. 2; [32, 38]), die einer modifizierten Hohl- und Luck-Klassifikation entspricht, ist auch bei der Schatzker-Klassifikation und der AO-Klassifikation zu beachten, dass diese Klassifikationssysteme anhand anterior-posteriorer Röntgenprojektionen entwickelt wurden. Infolgedessen sind sie, was ihre Aussagekraft hinsichtlich aller - auch komplexer - Frakturkonfigurationen angeht, insgesamt limitiert [31]. Bei der Überprüfung der Schatzker- und der AO-Klassifikation konnten verschiedene Studien nach Ergänzung der konventionellen Röntgenprojektion durch CT-Bildgebung inklusive 3-DRekonstruktionen eine höhere Intraund Interbeobachterreliabilität nachwei- 


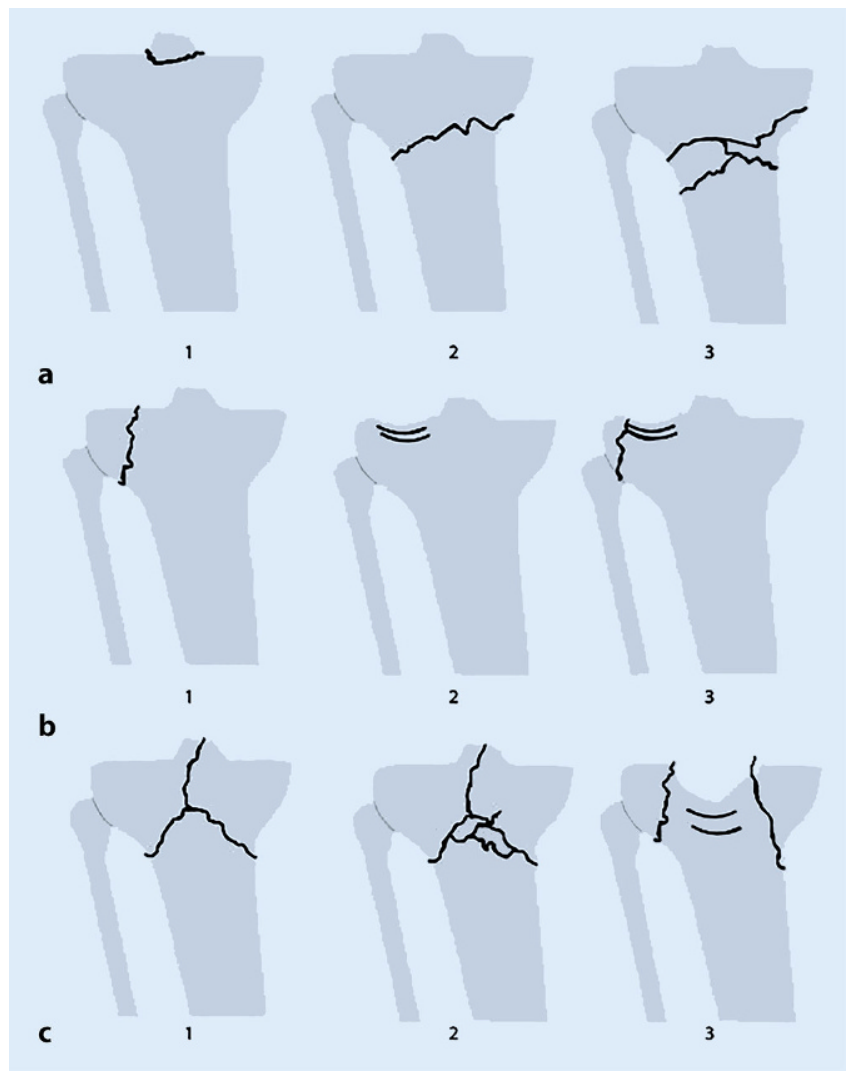

Abb. 1 A AO-Klassifikation der proximalen Tibiafraktur. a Knöcherne Ligamentausrisse und metaphysäre A-Frakturen, b unikondyläre, intraartikuläre Spalt-, Impressions- sowie unikondylären Kombinationsfrakturen, c bikondyläre bzw.Trümmerfrakturen. Die Einteilung $(1,2,3)$ beschreibt den Grad der Fragmentierung. AO Arbeitsgemeinschaft Osteosynthesefragen. (Aus [3], mod. nach [38])

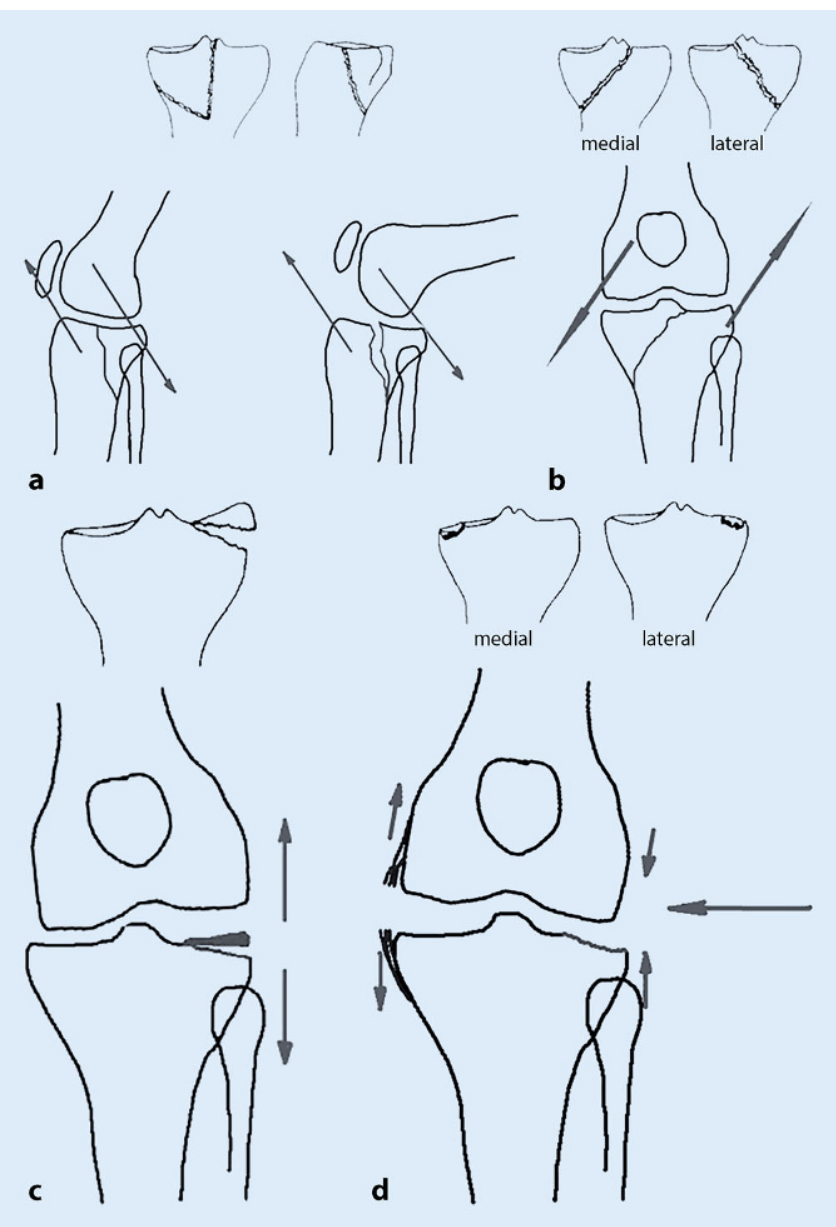

Abb. 2 ^ Moore-Klassifikation mit Verletzungsmechanismus: a Typ-I-Fraktur; b Typ-II-Fraktur; c Typ-III-Fraktur; d Typ-IV-Fraktur. (Aus [3]) sen. Für die Hohl- und Luck-Klassifikation dagegen ergab sich zwar unter Anwendung sowohl der konventionellen Bildgebung als auch der CT eine deutlich bessere Inter- und Intrabeobachterreliabilität als für die SchatzkerKlassifikation oder die AO-Klassifikation, allerdings hatte diese Klassifikation einige wesentliche Limitierungen hinsichtlich ihrer Fähigkeit, alle Frakturmuster ausreichend darzustellen [4, 7, 31]. In der klinischen Praxis erscheint unter Berücksichtigung ligamentärer und neurovaskulärer Begleitverletzungen die Unterteilung in Tibiaplateauund Tibiakopfluxationsfrakturen weiterhin sinnvoll. Die AO-Klassifikation stellt insbesondere die Plateaufrakturen dar, während die Moore-Klassifikation die Luxationsfrakturen mit potenziell begleitenden Weichteilverletzungen präziser abbildet. Überschneidungen beider Klassifikationen sind dabei vorhanden.

\section{„Moderne“ CT-basierte Klassifikationen}

Neben verschiedenen Klassifikationen, die als Modifikationen der oben dargestellten „klassischen“ Klassifikationssysteme bezeichnet werden können [12, 22], bestehen mittlerweile „modernere“ Klassifikationen, die die CT-Bildgebung inklusive 3-D-Rekonstruktionen berücksichtigen [28, 36]. Deren Entwicklung beruhte umgekehrt auf der mittlerweile vorhandenen Verfügbarkeit moderner winkelstabiler Plattensysteme, die die klinischen Ergebnisse nach operativer Therapie proximaler Tibiafrakturen nachhaltig verbessert haben [20]. Unsere Murnauer Arbeitsgruppe hat in den vergangenen Jahren untersucht, warum sich dennoch ein relativ hoher Prozentsatz sekundärer Osteosyntheseversagens nach offener Reposition und winkelstabiler Plattenosteosynthese bei proximalen Tibiafrakturen findet. Als Hauptgrund wurde ermittelt, dass die meisten verfügbaren winkelstabilen Plattensysteme für proximale Tibiafrakturen die Frakturlinien in der koronaren Ebene nicht suffizient erfassen [14], obwohl die Inzidenz großer koronarer Frakturlinien bei komplexen Tibiaplateaufrakturen mit bis zu $40 \%$ angegeben wird [2].

\section{) Bis zu $40 \%$ der komplexen proximalen Tibiafrakturen haben eine koronare Frakturlinie}

Koronare Frakturlinien sind jedoch in den gewöhnlich angewendeten Klassifikationen wie der Schatzker-Klassifikation und der AO-Klassifikation nicht abgebildet, da diese entwickelt wurden, als dreidimensionale CT-Rekonstruktionen noch nicht in der Breite verfügbar waren. 
Infolgedessen hat unsere Arbeitsgruppe kürzlich ein valides und reliables Klassifikationssystem für komplexe bikondyläre proximale Tibiafrakturen auf Basis einer systematischen Analyse der Frakturlinien in einem reproduzierbaren dreidimensionalen Frakturmodell erstellt.

\section{Spezifika der CT-basierten Klassifikation unter besonderer Berücksichtigung der koronaren Schnittbildebene}

Obwohl in neueren Studien versucht wurde, das Tibiaplateau genau zu kartographieren, um anschließend die Frakturen mit einem frakturspezifischen chirurgischen Zugang adressieren zu können, haben die Erkenntnisse dieser Analysen bisher noch nicht in ein systematisches Klassifikationssystem bzw. einen allgemein gültigen Behandlungsalgorithmus Einzug gehalten [28]. Im Gegensatz zu den CT-basierten Klassifikationssystemen, welche die Frakturmorphologie aus der axialen Schnittebene ableiten, nutzt die Einteilung in 3 morphologische Frakturtypen auch die koronaren und sagittalen Schnittebenen aus der 3-DBildgebung $[28$, 36]. Die in Murnau entwickelte CT-adaptierte und chirurgisch orientierte Klassifikation für komplexe bikondyläre proximale Tibiafrakturen, für die eine bessere Interbeobachterreliabilität als für die „klassischen“ AObzw. Schatzker-Klassifikationen ermittelt werden konnte, definiert 3 morphologische Typen und orientiert sich am Vorhandensein und dem Verlauf einer koronaren Frakturlinie (- Abb. 3), die mit einem großen dorsomedialen Fragment assoziiert ist [36].

\section{》) Koronare Frakturlinien resul- tieren in einem dorsomedialen Fragment}

Frühere Studien haben gezeigt, dass bei Vorliegen einer koronaren Frakturlinie eine zusätzliche mediale Plattenosteosynthese erforderlich ist. In unseren Untersuchungen konnten wir weiterhin nachweisen, dass sagittale Frakturlinien typischerweise in 2 Regionen lokalisiert sind, nämlich an der Eminentia

Trauma Berufskrankh 2018 $20: 230-236$ https://doi.org/10.1007/s10039-018-0400-0

(c) Der/die Autor(en) 2018

C. von Rüden · S. Samsami · R. Pätzold · P. Augat

Proximale Tibiafrakturen. Klassifikationen und biomechanische Grundlagen - ein Update

\section{Zusammenfassung}

Obwohl auch die Computertomographie(CT)basierten neueren Klassifikationen für proximale Tibiafrakturen nicht alle Frakturtypen exakt abbilden können, lässt sich aus ihnen ein individuelles Behandlungskonzept ableiten. Ihre Anwendung ermöglicht zudem einen strukturierten Zugang zur operativen Stabilisierung der meisten Frakturmuster. Eigene Studien konnten zeigen, wie wichtig die Beachtung der koronaren und sagittalen Frakturlinien ist. Es ist zu erwarten, dass die konsequente Anwendung reproduzierbarer dreidimensionaler CT-basierter Klassifikationen zu einem standardisierten operativen Behandlungsprotokoll führen und die präoperative Planung erleichtern wird, um so die Operationszeit zu reduzieren und die klinischen Resultate zu verbessern. Für die Stabilisierung unikondylärer Frakturen haben sich Kombinationen von Schraubenund Plattenosteosynthesen bewährt, die bei Impressionsfrakturen durch eine Verbundosteosynthese ergänzt werden können. Aufgrund ihrer Komplexität gibt es bei bikondylären Frakturen ein breites Spektrum an Osteosynthesemöglichkeiten. Wesentlich sind die Berücksichtigung der Frakturmorphologie und die stabile Retention der wesentlichen Frakturfragmente, insbesondere der dorsalen Frakturanteile.

Schlüsselwörter

Osteosynthese - Frakturtypen · Computertomographie - Behandlungsprotokoll . Präoperative Planung

\section{Proximal tibial fractures. Classifications and biomechanical principles-an update}

\section{Abstract}

Although newly developed computed tomography (CT)-based classifications for proximal tibial fractures could not exactly depict all fracture types, an individual treatment concept can be deduced from them. The use of these classifications enables a well-structured approach to stabilize most fracture patterns. In own studies it could be demonstrated how important it is to pay attention to coronal and sagittal fracture lines. Therefore, consistent use of reproducible three-dimensional CT-based classifications may lead to a standardized surgical treatment algorithm and may facilitate preoperative planning. Consequently, this may result in reduced operation times and improved clinical results. Unicondylar fractures can be successfully stabilized by the combination of screw and plate osteosynthesis, which in the case of impression fractures of the tibial plateau has to be supplemented by additional defect augmentation. Due to the complexity of bicondylar fractures there is a broad spectrum of options available for osteosynthesis. It is essential to make an accurate analysis of fracture morphology and the stable retention of essential fracture fragments, particularly the dorsal fragments.

\section{Keywords}

Osteosynthesis - Fracture type - Computed tomography · Treatment protocol · Preoperative planning intercondylaris und am lateralen Anteil des lateralen Tibiaplateaus (-Abb. 3): Frakturen des Typs I involvieren die Gelenkfläche der Eminentia und des lateralen Kondylus, ohne die mediale Gelenkfläche zu treffen (ohne koronare Frakturlinie). Frakturen des Typs II betreffen den medialen Kondylus ohne einen Hauptfrakturlinienwinkel zwischen 60 und $120^{\circ}$ und schließen die mediale Gelenkfläche ein. Frakturen des
Typs III betreffen die mediale Gelenkfläche mit einem Hauptfrakturlinienwinkel zwischen 60 und $120^{\circ}$ (koronare Frakturlinie).

Die strukturierte Anwendung dieser Klassifikation an einer großen Anzahl von Frakturen im eigenen Patientengut in einem Beobachtungszeitraum von 5 Jahren ergab Frakturen des Typs 1 bei $30 \%$, des Typs 2 bei $23 \%$ und des Typs 3 bei $47 \%$ der Untersuchten. Sie eröffnete die Mög- 


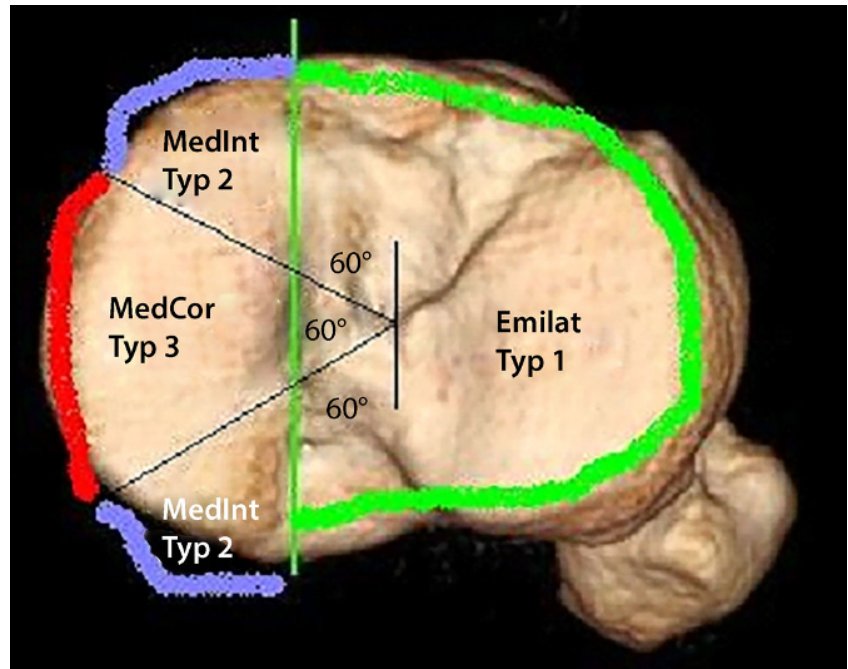

Abb. 3 A Die Computertomographie-adaptierte und chirurgisch orientierte Klassifikation für komplexe bikondyläre proximale Tibiafrakturen definiert 3 morphologische Typen und orientiert sich am Vorhandensein und dem Verlauf einer koronaren Frakturlinie. Typ 1: Frakturen, die sich nur auf die laterale Kondyle und die Eminentia beschränken. Typ 2: Frakturen, die eine Mitbeteiligung der medialen Gelenkfläche haben, aber deren Winkel kleiner als $60^{\circ}$ oder größer als $120^{\circ}$ ist. Typ 3: Frakturen mit einer Verletzung der medialen Kondyle, deren Winkel $60-120^{\circ}$ beträgt. (Aus [36], mit freundl. Genehmigung von Elsevier)

lichkeit, eine direkte chirurgische Therapieempfehlung wie folgt abzugeben:

- Für Frakturen des Typs I ist die isolierte Plattenosteosynthese von lateral ausreichend.

- Frakturen des Typs II erfordern die zusätzliche Stabilisierung des medialen Kondylus mit medialer Abstützplatte.

- Für Frakturen des Typs III wird die zusätzliche Stabilisierung des medialen Tibiaplateaus in anteriorposteriorer oder posterior-anteriorer Richtung empfohlen [36].

Diese Klassifikation lässt zudem ebenso wie die kürzlich von Luo vorgestellte CTbasierte 3-Säulen-Klassifikation für proximale Tibiafrakturen [29] eine exakte Planung des optimalen operativen $\mathrm{Zu}$ ganges und der korrekten Positionierung des Osteosynthesematerials zu. Obwohl sich beide Klassifikationen nicht auf alle Frakturtypen anwenden lassen, ermöglichen sie einen strukturierten Zugang zu den wesentlichen Frakturmustern.

\section{Biomechanik}

Die Wiederherstellung der Funktion des Kniegelenks ist das wesentliche Ziel bei der Behandlung von Frakturen der pro- ximalen Tibia. Hierzu gehören die Wiederherstellung der mechanischen Belastungsfähigkeit und der physiologischen Kniegelenkbeweglichkeit. Die mechanische Belastung des Tibiaplateaus während des Gehens entspricht etwa dem 3-fachen Körpergewicht und teilt sich im Verhältnis 2 zu 3 zwischen dem lateralen und dem medialen Plateau auf. Bei stärker belastenden Aktivitäten, wie z. B. Treppensteigen, steigt die Belastung bis zum 4-fachen Körpergewicht an. Die Belastungen sind hauptsächlich axial in Richtung der Achse des Tibiaschafts orientiert. Die Kräfte in anteroposteriorer und mediolateraler Richtung betragen bis zum 0,3-fachen des Körpergewichts, wobei die höchsten Kräfte in anteroposteriorer Richtung bei der Kniebeuge auftreten [40].

\section{Frakturmechanismus}

Frakturen der proximalen Tibia entstehen entweder durch axiale Krafteinwirkung auf das Tibiaplateau (Stauchungsbrüche), durch Krafteinwirkung auf das Kniegelenk mit Hebelwirkung (Biegungsbrüche in varus oder valgus) oder durch Kombinationsbelastungen. Durch Rotationsbelastungen um die Schaftachse entstehen eher Verletzungen am Bandapparat und seltener Torsionsbrüche. Stauchungsbrüche manifestieren sich als Impressions- oder Spaltbrüche des Tibiaplateaus und führen zu Schädigungen des Knorpels am Tibiaplateau und oft auch zu Meniskusläsionen. Biegungsbrüche resultieren häufig in einer Depression des Tibiaplateaus auf der der Krafteinwirkung zugewandten Seite und sind in aller Regel mit Verletzungen des Bandapparates im Kniegelenk assoziiert. Während bei jüngeren Menschen aufgrund des Unfallmechanismus und der guten Knochenqualität meist Spaltbrüche $\mathrm{zu}$ beobachten sind, treten mit zunehmendem Patientenalter häufiger Impressions- oder Depressionsfrakturen auf.

\section{》) Frakturen des Tibiaplateaus treten ab Kräften von $6000 \mathrm{~N}$ auf}

Die Kräfte, die zum Bruch des Tibiaplateaus führen, betragen bei axialer Krafteinleitung etwa zwischen 6000 und $8000 \mathrm{~N}$, entsprechend dem 8- bis 10fachen Körpergewicht [11]. In Flexionsstellung des Kniegelenks können Frakturen des Tibiaplateaus bereits bei Lasten ab $5000 \mathrm{~N}$, die axial auf das Plateau wirken, auftreten $[1,19]$.

\section{Frakturstabilisierung}

Ziele der operativen Frakturbehandlung an der proximalen Tibia sind, wie in der Einleitung angesprochen, die akkurate Wiederherstellung der Gelenkflächenkongruenz und des korrekten Alignements (Reposition) sowie die stabile Fixierung der Fragmente durch eine Osteosynthese (Retention). Bei Beteiligung der Gelenkfläche ist in der Regel ein offenes Vorgehen zur Erzielung einer adäquaten Reposition erforderlich („open reduction and internal fixation“ [ORIF]). Dadurch kann die Gelenkfläche erhalten und das Risiko einer posttraumatischen Arthrose reduziert werden. Für das korrekte Alignement ist zu beachten, dass die Gelenkfläche des Tibiaplateaus nicht mit der Achse des Tibiaschafts fluchtet, sondern nach dorsal übersteht. Weiterhin ist das Tibiaplateau von ventral nach dorsal absteigend (engl. ,slope“) und als 
Ausdruck der Reklination des proximalen Tibiaendes um bis zu $7^{\circ}$ verkippt. Für die Retention ist auf die dislozierende Wirkung der kniegelenkübergreifenden Muskulatur zu achten. Über den Ansatz der Patellarsehne an der Tuberositas tibiae wirkt der M. quadriceps femoris bei Kontraktion nach proximal und anterior dislozierend. Dislokationen an den seitlichen Gelenkflächen können durch den M. sartorius und den M. gracilis (medial) sowie den M. tibialis anterior (anterolateral) provoziert werden. Posterior erzeugen die hinter der Kniegelenkachse verlaufende Beugemuskulatur des Oberschenkels und die beiden Köpfe des M. gastrocnemius dislozierende Kräfte [15].

Der Erhalt der Retention wird durch die Fixierung der Fraktur gewährleistet. Für eine erfolgreiche Heilung der epiund metaphysären Frakturanteile ist eine mechanisch stabile Osteosynthese obligatorisch. Diese gewährleistet die Initiierung des Frakturheilungsprozesses und ermöglicht eine frühe Mobilisierung des Kniegelenks. Bei stabiler Osteosynthese kann die frühzeitige Lockerung des Osteosynthesematerials vermieden werden.

\section{Osteosyntheseverfahren}

Vergleichende Studien zur biomechanischen Evaluation von Osteosyntheseverfahren proximaler Tibiafrakturen haben aus den oben erwähnten Gründen meist zum Ziel, das stabilere Fixierungsverfahren zu identifizieren. Dazu wird die initiale Stabilität der Osteosynthese über die Bestimmung der Steifigkeit des Konstrukts aus Implantat und Knochen bestimmt. Eine hohe Steifigkeit der Osteosynthese bedeutet dabei eine hohe Stabilität der Fixierung, verbunden mit einer guten Aussicht auf eine erfolgreiche Frakturheilung. Weiterhin wird die Zeitstabilität der Osteosynthese in zyklischen Dauertests ermittelt. Damit soll einerseits das Risiko für die Lockerung des Implantats im Knochen und andererseits das Risiko eines Osteosyntheseversagens durch Platten- oder Schraubenbruch oder durch Schraubenlockerung bestimmt werden.

Für die osteosynthetische Stabilisierung proximaler Tibiafrakturen stehen in erster Linie konventionelle und winkelstabile Plattensysteme sowie intramedulläre Nägel zur Verfügung. Plattenosteosynthesen vereinfachen die Frakturreposition im Vergleich zur Marknagelosteosynthese, da v. a. anatomisch präformierte Platten als Leitschiene für die Frakturfragmente dienen können. Bei einfacheren Frakturmustern mit extraartikulärer Schaftkomponente stehen moderne Marknagelsysteme optional zur Verfügung. Durch die Kombination von Zugbzw. Kompressionsschrauben mit einem intramedullären Kraftträger können diese Frakturen ausreichend stabilisiert werden. Im Vergleich zur Osteosynthese mit einer lateral angebrachten winkelstabilen Platte können eine gleichwertige Belastungsfestigkeit und durch die zentrale Lage des intramedullären Kraftträgers sogar eine höhere Steifigkeit des Konstrukts erreicht werden [20]. Nichtsdestotrotz bleibt die intraartikuläre proximale Tibiafraktur nach wie vor eine Domäne der Plattenosteosynthese. Ergänzt werden kann diese durch überwiegend temporär verwendete externe Fixateure.

Einfache Frakturen des lateralen Tibiaplateaus (laterale Spaltfrakturen) können durch eine Kompressionsosteosynthese mit 2 oder 3 Zugschrauben ausreichend stabil fixiert werden. Dabei ist die Verwendung der dritten Schraube nicht notwendigerweise mit einer relevanten Verbesserung der mechanischen Stabilität der Fraktur verknüpft [33, 35]. Auch die zusätzliche Verwendung einer lateralen Abstützplatte erbringt nur geringfügige Verbesserungen der Zeitstabilität dieser lateralen Spaltfrakturen [27]. Erst bei einem größeren Frakturfragment oder einer mehrfragmentären Fraktur sollte für den Erhalt der Stabilität eine laterale Abstützplatte verwendet werden. Diese komprimiert das Fragment zurück an seine ursprüngliche Position, verhindert so das Abrutschen des Fragments nach distal und sichert die Abstützung des distalen Fragmentendes auf der intakten Kortikalis. Dabei sollte keine winkelstabile Platte verwendet werden, da diese die Kompression des Fragments nicht ausreichend gewährleistet [37].

Bei lateralen Spalt-/Depressions- oder Impressionsfrakturen, die mit zunehmendem Alter häufiger beobachtet wer- den, muss das imprimierte Fragment aufgerichtet und stabilisiert werden. Die passgenaue Aufrichtung des Fragments verbessert die Langzeitstabilität der Osteosynthese und ist daher eine wesentliche Grundvoraussetzung für die erfolgreiche Frakturstabilisierung [17]. Für die Osteosynthese stehen verschiedene Kombinationen von Osteosyntheseverfahren mit Platten, Kortikalis- oder Spongiosaschrauben sowie autologem oder künstlichem Knochenersatz zur Verfügung. Wesentlich für eine biomechanisch stabile Fixierung erscheint die Abstützung des eingedrückten Fragments durch stegförmig angeordnete subchondrale Schrauben [26]. Diese sollten - außer bei besonders guter Knochenqualität - durch eine lateral angelegte Platte abgesichert werden. Die Schrauben können dabei oberhalb der Platte oder durch die Platte platziert werden, wobei die Platzierung durch die Platte geringfügige Vorteile für die Zeitstabilität des Konstrukts zu haben scheint [5]. Eine sog. „Verbundosteosynthese" mit zusätzlicher Augmentation des eingedrückten Fragments verbessert grundsätzlich die mechanische Stabilität. Während die Verwendung eines Autografts die Heilung der Fraktur am besten unterstützt, erweist sich die Verwendung von allogenem Knochenersatzmaterial als biomechanisch vorteilhafte Alternative [42].

Frakturen des medialen Tibiaplateaus sind meist auf eine große Krafteinwirkung bzw. ein Hochrasanztrauma zurückzuführen und resultieren in Dislokationen, Bandverletzungen und auch Nerven- und Gefäßschädigungen. Sowohl einfache, v. a. aber mehrfragmentäre Frakturen benötigen zur adäquaten Stabilisierung eine mediale Abstützplatte. Bei großen Fragmenten oder sehr instabilen Situationen kann eine sog. "Doppelplattenosteosynthese“ (anteromedial und posteromedial) eine deutliche Erhöhung der Frakturstabilität erreichen. Eine Plattenosteosynthese nur von lateral selbst mit langen Schrauben in winkelstabiler Technik kann das mediale Plateau dagegen nicht ausreichend stabilisieren [25]. Durch die laterale Plattenlage können weder die Platte noch die Schrauben eine ausreichende 
Kompression der Fraktur erreichen und bieten daher keinen Schutz gegen Scherbelastungen, v. a. da der Frakturverlauf meist in der koronaren Ebene und damit parallel zu den Schrauben ausgerichtet ist. Bei posterior gelegenen Frakturen kann auch eine nicht notwendigerweise winkelstabile Plattenosteosynthese von posteromedial eine ausreichende mechanische Stabilität herstellen, da sie sich gegen die intakte posteriore Kortikalis abstützen kann [43].

\section{》) Bikondyläre proximale Tibiafrakturen erfordern die stabile Fixierung des medialen Fragments}

Bikondyläre Frakturen der proximalen Tibia besitzen die höchste Komplexität und erfordern eine sorgfältige präoperative Planung. Wegen der großen Bandbreite der Frakturausprägungen gibt es zu den bikondylären Frakturen auch die meisten biomechanischen Untersuchungen. Die meisten dieser Studien beruhen auf dem von Horwitz vorgestellten Modell der bikondylären Tibiafraktur mit diaphysärer Fraktur (Typ Schatzker VI, AO 41 C 3). Dabei werden der laterale und der mediale Kondylus durch einen in der Sagittalebene schräg verlaufenden Bruch, ausgehend von der Eminentia intercondylaris, abgetrennt. Das dadurch erhaltene Frakturmuster entspricht in der anterior-posterioren Projektion dem typischen klinischen Bild einer bikondylären Tibiafraktur. Für die Übertragbarkeit des Modells muss beachtet werden, dass es keine koronare Frakturebene erhält, obwohl diese bei bis zu $40 \%$ aller bikondylären Tibiafrakturen in der CT nachweisbar ist [2, 41] Basierend auf dem von Horwitz [23] eingeführten biomechanischen Frakturmodell, kommen die meisten Studien zu der Schlussfolgerung, dass bikondyläre Frakturen adäquat mit einer winkelstabilen Platte von lateral fixiert werden können. Horwitz selbst [23] zeigte noch in der Zeit, bevor winkelstabile Implantate an der Tagesordnung waren, dass eine lateral angebrachte $\mathrm{Ab}$ stützplatte ohne Winkelstabilität deutlich größere Fragmentbewegungen zulässt als eine „Doppelplattenosteosynthese“ und daher keine ausreichende Stabilität erreichen kann. Nur 4 Jahre später konnten Mueller at al. nachweisen, dass eine lateral angebrachte winkelstabile Platte zwar geringfügig mehr Fragmentbewegungen zulässt als eine „Doppelplattenosteosynthese" mit konventioneller Platte, aber die gleiche Konstruktsteifigkeit und -festigkeit aufweist [34]. Diese Ergebnisse konnten sowohl in biomechanischen Untersuchungen als auch in klinischen Beobachtungen bestätigt werden $[10,13]$. Genauere Untersuchungen der Bewegungen der einzelnen Fragmente einer bikondylären proximalen Tibiafraktur lassen allerdings Defizite einer rein lateralen Plattenosteosynthese gegenüber einem Konstrukt mit zusätzlicher medialer Platte erkennen. Wie zu erwarten, treten die Defizite der Stabilisierung v. a. am medialen Fragment auf [18]. Um die Instabilität des medialen Fragments zu reduzieren, kann durch geeignete Wahl der Schraubenlänge eine Abstützung der proximalen Schrauben an der medialen Kortikalis erreicht werden. Sowohl die Konstruktsteifigkeit als auch deren Versagenslast können durch diese Maßnahme effektiv um bis zu $80 \%$ erhöht werden [8].

Die technischen Fortschritte in der Entwicklung winkelstabiler Plattensysteme wurden auch hinsichtlich ihrer Anwendung an der proximalen Tibia getestet. So zeigten weniger stark auftragende Platten $(3,5 \mathrm{~mm})$ zumindest bei stabilen Knochenverhältnissen eine ähnliche Stabilität wie Großfragmentplatten $(4,5 \mathrm{~mm})$ [16]. Auch polyaxiale Winkelverbindungen scheinen genügend Stabilität für die Fixierung bikondylärer Tibiafrakturen aufzuweisen und stehen festen Winkelverbindungen unter der Voraussetzung korrekten Einbringens hinsichtlich der mechanischen Stabilität in nichts nach [6].

\section{Fazit für die Praxis}

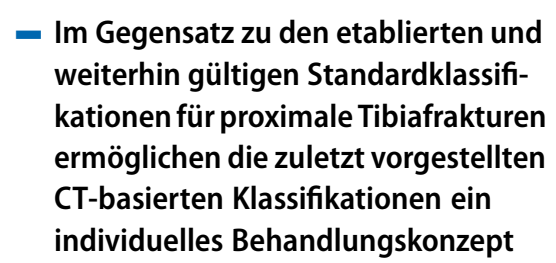

und einen strukturierten Zugang zur operativen Stabilisierung der wesentlichen Frakturmuster.

- Die konsequente Anwendung und zunehmende Etablierung moderner dreidimensionaler CT-basierter Klassifikationen wird erwartungsgemäß in Zukunft die präoperative Planung weiter erleichtern und zu einem standardisierten Behandlungsalgorithmus führen.

- Basierend auf der akkuraten Frakturklassifikation lässt sich das unter biomechanischen Gesichtspunkten am besten geeignete Osteosyntheseverfahren auswählen. In Kombination mit optimierten Operationstechniken und Osteosynthesematerialien lassen sich die klinischen Resultate zukünftig weiter verbessern.

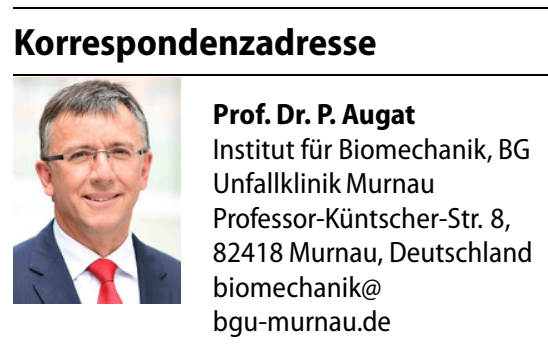

Funding. Open access funding provided by Paracelsus Medical University.

\section{Einhaltung ethischer Richtlinien}

Interessenkonflikt. C. von Rüden, S. Samsami, R. Pätzold und P. Augat geben an, dass kein Interessenkonflikt besteht.

Dieser Beitrag beinhaltet keine von den Autoren durchgeführten Studien an Menschen oder Tieren.

Open Access Dieser Artikel wird unter der Creative Commons Namensnennung 4.0 International Lizenz (http://creativecommons.org/licenses/by/4.0/deed. de) veröffentlicht, welche die Nutzung, Vervielfältigung, Bearbeitung, Verbreitung und Wiedergabe in jeglichem Medium und Format erlaubt, sofern Sie den/die ursprünglichen Autor(en) und die Quelle ordnungsgemäß nennen, einen Linkzur Creative Commons Lizenz beifügen und angeben, ob Änderungen vorgenommen wurden.

\section{Literatur}

1. Banglmaier RF, Dvoracek-Driksna D, Oniang'o TE, Haut RC (1999) Axial compressive load response of the $90^{\circ}$ flexed human tibiofemoral joint. In: Stapp car crash conference proceedings. Society of Automotive Engineers, San Diego 
2. Barei DP, O'mara TJ, Taitsman LA et al (2008) Frequency and fracture morphology of the posteromedial fragment in bicondylar tibial plateau fracture patterns. J Orthop Trauma 22:176-182

3. Bobrich E, Haupt C, Grass R, Zwipp H (2009) Tibial plateau fractures and luxation fractures. Fracture classification and associated lesions. Trauma Berufskrankh 11(Suppl 2):154-159

4. Brunner A, Horisberger M, Ulmar B et al (2010) Classification systems for tibial plateau fractures; does computed tomography scanning improve their reliability? Injury 41:173-178

5. Cross WW 3rd, Levy BA, Morgan JA et al (2013) Periarticular raft constructs and fracture stability in split-depression tibial plateau fractures. Injury 44:796-801

6. Cullen AB, Curtiss S, Lee MA (2009) Biomechanical comparison of polyaxial and uniaxial locking plate fixation in a proximal tibial gap model. J Orthop Trauma 23:507-513

7. Doornberg JN, Rademakers MV, Van Den Bekerom MP et al (2011) Two-dimensional and threedimensional computed tomography for the classification and characterisation of tibial plateau fractures. Injury 42:1416-1425

8. Dougherty PJ, Kim DG, Meisterling S et al (2008) Biomechanical comparison of bicortical versus unicortical screw placement of proximal tibia locking plates: a cadaveric model. J Orthop Trauma 22:399-403

9. Duparc J, Ficat P (1960) Articular fractures of the upper end of the tibia. Rev Chir Orthop Reparatrice Appar Mot 46:399-486

10. Egol KA, Su E, Tejwani NC et al (2004) Treatment of complex tibial plateau fractures using the less invasive stabilization system plate: clinical experience and a laboratory comparison with double plating. J Trauma 57:340-346

11. Funk JR, Tourret LJ, Crandall JR (2000) Experimentally produced tibial plateau fractures. International Research Council on the Biomechanics of Injury, Conference, Montpellier, S 171-182

12. Gicquel T, Najihi N, Vendeuvre T et al (2013) Tibial plateau fractures: reproducibility of three classifications (Schatzker, AO, Duparc) anda revised Duparc classification. Orthop Traumatol Surg Res 99:805-816

13. Gosling T, Schandelmaier P, Marti A et al (2004) Less invasive stabilization of complex tibial plateau fractures: a biomechanical evaluation of a unilateral locked screw plate and double plating. JOrthop Trauma 18:546-551

14. Gosling T, Schandelmaier P, Muller M et al (2005) Single lateral locked screw plating of bicondylar tibial plateau fractures. Clin Orthop Relat Res 439:207-214

15. Hansen M, Mehler D, Voltmer W et al (2002) The extraarticular proximal tibial fractures. Unfallchirurg 105:858-872

16. Hasan S, Ayalon OB, Yoon RS et al (2014) A biomechanical comparison between locked 3.5$\mathrm{mm}$ plates and $4.5-\mathrm{mm}$ plates for the treatment of simple bicondylar tibial plateau fractures: is bigger necessarily better? JOrthop Traumatol 15:123-129

17. Heiney JP, Kursa K, Schmidt AH et al (2014) Reduction and stabilization of depressed articular tibial plateau fractures: comparison of inflatable and conventional bone tamps: study of a cadaver model. JBone Joint Surg Am 96:1273-1279

18. Higgins TF, Klatt J, Bachus KN (2007) Biomechanical analysis of bicondylar tibial plateau fixation: how does lateral locking plate fixation compare to dual plate fixation? J Orthop Trauma 21:301-306
19. Hirsch G, Sullivan L (1965) Experimental knee-joint fractures. A preliminary report. Acta Orthop Scand 36:391-399

20. Hogel F, Hoffmann S, Panzer S et al (2013) Biomechanical comparison of intramedullar versus extramedullar stabilization of intra-articular tibial plateau fractures. Arch Orthop Trauma Surg 133:59-64

21. Hohl M, Luck JV (1956) Fractures of the tibial condyle; a clinical and experimental study. J Bone Joint Surg Am 38-A:1001-1018

22. Honkonen SE, Jarvinen MJ (1992) Classification of fractures of the tibial condyles. J Bone Joint Surg Br 74:840-847

23. Horwitz DS, Bachus KN, Craig MA et al (1999) A biomechanical analysis of internal fixation of complex tibial plateau fractures. J Orthop Trauma 13:545-549

24. Hu YL, Ye FG, Ji AY et al (2009) Three-dimensiona computed tomography imaging increases the reliability of classification systems for tibial plateau fractures. Injury 40:1282-1285

25. Jiang R, Luo CF, Zeng BF (2008) Biomechanical evaluation of different fixation methods for fracture dislocation involving the proximal tibia. Clin Biomech (Bristol, Avon) 23:1059-1064

26. Karunakar MA, Egol KA, Peindl R et al (2002) Split depression tibial plateau fractures: a biomechanical study. J Orthop Trauma 16:172-177

27. Koval KJ, Polatsch D, Kummer FJ et al (1996) Split fractures of the lateral tibial plateau: evaluation of three fixation methods. J Orthop Trauma 10:304-308

28. Krause M, Preiss A, Muller G et al (2016) Intraarticular tibial plateau fracture characteristics according to the "ten segment classification". Injury 47:2551-2557

29. Luo CF, Sun H, Zhang B et al (2010) Three-column fixation for complex tibial plateau fractures. J Orthop Trauma 24:683-692

30. Marsh JL, Slongo TF, Agel J et al (2007) Fracture and dislocation classification compendium-2007: Orthopaedic Trauma Association classification, database and outcomes committee. J Orthop Trauma 21:S1-S133

31. Millar SC, Arnold JB, Thewlis D et al (2018) A systematic literature review of tibial plateau fractures: What classifications are used and how reliable and useful are they? Injury 49:473-490

32. Moore TM, Patzakis MJ, Harvey JP (1987) Tibial plateau fractures: definition, demographics, treatment rationale, and long-term results of closed traction management or operative reduction. JOrthop Trauma 1:97-119

33. Moran E, Zderic I, Klos Ket al (2017) Reconstruction of the lateral tibia plateau fracture with a third triangular support screw: a biomechanical study. JOrthop Translat 11:30-38

34. Mueller $\mathrm{KL}$, Karunakar MA, Frankenburg EP et al (2003) Bicondylar tibial plateau fractures: a biomechanical study. Clin Orthop Relat Res 412:189-195

35. Parker PJ, Tepper KB, Brumback RJ et al (1999) Biomechanical comparison of fixation of type-I fractures of the lateral tibial plateau. Is the antiglide screw effective? JBone Joint Surg Br 81:478-480

36. Patzold R, Friederichs J, Von Ruden C et al (2017) The pivotal role of the coronal fracture line for a new three-dimensional CT-based fracture classification of bicondylar proximal tibial fractures. Injury 48:2214-2220

37. Rahm S, Ebneter L, Schultz B et al (2016) Comparison of interfragmentary compression in conventional and locked plating of proximal unicondylar tibia fractures: a biomechanical cadaver study. Acta Orthop Belg 82:586-592

38. Raschke M, Zantop T, Petersen W (2007) Fracture of the tibial head. Chirurg 78:1157-1169 (quiz 1170-1151)

39. Schatzker J, Mcbroom R, Bruce D (1979) The tibial plateau fracture. The Toronto experience 1968-1975. Clin Orthop Relat Res 138:94-104

40. Taylor WR, Schutz P, Bergmann G et al (2017) A comprehensive assessment of the musculoskeletal system: the CAMS-knee data set. J Biomech 65:32-39

41. Weaver MJ, Harris MB, Strom AC et al (2012) Fracture pattern and fixation type related to loss of reduction in bicondylar tibial plateau fractures. Injury 43:864-869

42. Welch RD, Zhang H, Bronson DG (2003) Experimental tibial plateau fractures augmented with calcium phosphate cement or autologous bone graft. J Bone Joint Surg Am 85-A:222-231

43. ZengZM,LuoCF,PutnisSetal(2011)Biomechanical analysis of posteromedial tibial plateau split fracture fixation. Knee 18:51-54 